\title{
A Note on Two (3+1)-Dimensional Gardner-Type Equation with Multiple Kink Solutions
}

\author{
Afgan Aslanov \\ Computer Engineering Department, Istanbul Esenyurt University, Istanbul, Turkey.
}

How to cite this paper: Afgan Aslanov. (2021) A Note on Two (3+1)-Dimensional Gardner-Type Equation with Multiple Kink Solutions. Journal of Applied Mathematics and Computation, 5(4), 354-360.

DOI: $10.26855 /$ jamc.2021.12.014

Received: November 15, 2021

Accepted: December 7, 2021

Published: December 23, 2021

*Corresponding author: Afgan Aslanov, Computer Engineering Department, Istanbul Esenyurt University, Istanbul, Turkey.

Email: afganaslan@esenyurt.edu.tr

\begin{abstract}
It is difficult or impossible to find the exact solutions for many partial differential equations. In recent years, a variety of efficient and practical methods have been proposed by mathematicians. This article investigates the exact solutions of partial differential equations. The Gardner equation is well known in literature and is applicable in various branches of physics. The Gardner equation belongs to the category of non-linear partial differential equations. This equation and its generalizations are used in many areas of applications, such as hydrodynamics, plasma physics, and quantum field theory. The Gardner-type equations are the useful model to understand the propagation of negative ion acoustic plasma waves. These type of equations can be derived from the system of plasma motion equations in one dimension with arbitrarily charged cold ions and inertia neglected isothermal electrons. Numerous numerical and analytical methods have been used to study this equation. That proves the importance of this equation. In this paper, we examine the exact travelling wave solutions of the Gardner equation. The Hirota's bilinear method and the Cole-Hopf transformation is used to obtain an elegant formula for the exact travelling wave solution. We demonstrate a correct formula for exact solutions. Mathematica software and the standart LaTex tools was used to perform the computations. The suggested approach can be used in other real-world models in science and engineering.
\end{abstract}

\section{Keywords}

Gardner-type equation, Hirota bilinear method, soliton solutions, kink solutions

\section{Introduction}

In the present paper by “Gardner equation”, we assume an equation in integro-differential form

$$
u_{t}+6 \beta u u_{x}+u_{x x x}-\frac{3}{2} \alpha^{2} u^{2} u_{x}+3 \sigma^{2} \partial_{x}^{-1} u_{y y}-3 \alpha \sigma u_{x} \partial_{x}^{-1} u_{y}=0,
$$

where $\sigma^{2}= \pm 1, \alpha, \beta$ are arbitrary constants and $\partial_{x}^{-1}$ is the inverse of $\partial_{x}$ with $\partial_{x}^{-1} \partial_{x}=\partial_{x} \partial_{x}^{-1}=1$. The equation (1) is widely used in plasma physics, fluid physics, quantum field theory and different branches of physics [1-7]. There are different methods, such as the inverse scattering method, the Casorati and Grammian determinant solutions, and the Hirota's direct method for solving equation (1) see, for example, [1,10]. For the travelling wave solutions of the Gardner-type equation in terms of corresponding ordinary differential equations see [12,13].

The more general forms of the equation (1) were examined in [11] by using the simplified Hirota's method:

$$
u_{t}+6 \beta \mathrm{u} u_{x}+u_{x x x}-\frac{3}{2} \alpha^{2} u^{2} u_{x}+3 \sigma^{2} \partial_{x}^{-1} u_{y y}-3 \alpha \sigma u_{x} \partial_{x}^{-1} u_{y}+3 \sigma^{2} \partial_{x}^{-1} u_{z z}=0,
$$




$$
\mathrm{u}_{\mathrm{t}}+6 \beta \mathrm{u} u_{x}+u_{x x x}-\frac{3 \alpha^{2} u^{2} u_{x}}{2}+3 \sigma^{2} \partial_{x}^{-1} u_{y y}-3 \alpha \sigma u_{x} \partial_{x}^{-1} u_{y}+3 \sigma^{2} \partial_{x}^{-1} u_{z z}-3 \alpha \sigma u_{x} \partial_{x}^{-1} u_{z}=0 .
$$

In this work, we analyze the equation (2) and find an elegant formula for the exact travelling wave solution.

\section{The travelling-wave solutions of the generalized Gardner-type equation}

In this Section, we first derive kink solutions for the Gardner-type equation (2), where

$$
\left(\partial_{x}^{-1} f\right)(x)=\int_{-\infty}^{x} f(t) d t,
$$

with decaying condition at infinity.

The transformation

$$
u(x, y, z, t)=v_{x}(x, y, z, t)
$$

translates (2) into the equation

$$
v_{x t}+6 \beta v_{x} v_{x x}+v_{x x x x}-\frac{3}{2} \alpha^{2} v_{x}^{2} v_{x x}+3 \sigma^{2} v_{y y}-3 \alpha \sigma v_{x x} v_{y}+3 \sigma^{2} v_{z z}=0
$$

To obtain the exact solution we substitute

$$
v(x, y, z, t)=e^{k x+r y+s z-c t}
$$

into the linear terms of (6), which gives $c$ as

$$
c=\frac{1}{k}\left(3 r^{2} \sigma^{2}+3 s^{2} \sigma^{2}+k^{4}\right),
$$

and the dispersion variable as

$$
\theta=k x+r y+s z-\frac{1}{k}\left(3 r^{2} \sigma^{2}+3 s^{2} \sigma^{2}+k^{4}\right) t .
$$

Next we use the Cole-Hopf transformation

$$
u(x, y, z, t)=R(\ln f(x, y, z, t))_{x}
$$

or

$$
v(x, y, z, t)=R(\ln f(x, y, z, t)) .
$$

To get the auxiliary function $f(x, y, z, t)$, we use the simplified Hirota's method: Substituting

$$
f(x, y, z, t)=1+e^{k x+r y+s z-\frac{1}{k}\left(3 r^{2} \sigma^{2}+3 s^{2} \sigma^{2}+k^{4}\right) t}
$$

into (6), [11] gives the single-kink solution:

$$
\begin{gathered}
\left.u(x, y, z, t)=v_{x}(x, y, z, t)\right) \\
=\frac{2 k e^{k x+\frac{k(2 \beta-\alpha k)}{\sigma \alpha} y+s z-\left(k^{3}+\frac{3 k(2 \beta-\alpha k)^{2}}{\alpha^{2}}+3 \alpha^{2 \frac{s^{2}}{k}}\right) t}}{\alpha\left(1+e^{k x+\frac{k(2 \beta-\alpha k)}{\sigma \alpha} y+s z-\left(k^{3}+\frac{3 k(2 \beta-\alpha k)^{2}}{\alpha^{2}}+3 \alpha^{2 \frac{s^{2}}{k}}\right) t}\right)},
\end{gathered}
$$

(Eq. (20) in [11]). The author [11] then used the same approach to establish the similar formula for the double-kink solution

$$
u=\frac{2 \sum_{i=1}^{2} k_{i} e^{k_{i} x+\frac{k_{i}\left(2 \beta-\alpha k_{i}\right)}{\sigma \alpha} y+s_{i} z-\left(k_{i}{ }^{3}+\frac{3 k_{i}\left(2 \beta-\alpha k_{i}\right)^{2}}{\alpha^{2}}+3 \alpha^{2} \frac{s_{i}^{2}}{k_{i}}\right) t}}{\alpha\left(1+\sum_{i=1}^{2} k_{i} e^{k_{i} x+\frac{k_{i}\left(2 \beta-\alpha k_{i}\right)}{\sigma \alpha} y+s_{i} z-\left(k_{i}{ }^{3}+\frac{3 k_{i}\left(2 \beta-\alpha k_{i}\right)^{2}}{\alpha^{2}}+3 \alpha \alpha^{2} \frac{s_{i}^{2}}{k_{i}}\right) t}\right)}
$$

Let us show that the formula (14) is not correct and prove the correct formula.

Example 2.1. Let $k_{1}=1, k_{2}=2, s_{1}=1, s_{2}=0, \alpha=2, \beta=\sigma=1$, it is easy to show that (14) is not the solution of the equation (6). Indeed 


$$
\begin{gathered}
u=\frac{2\left(e^{x+\frac{(2-2)}{2} y+z-\left(1+\frac{\left.3(2-2)^{2}\right)}{4}+3 \frac{1}{1}\right) t}+2 e^{2 x+\frac{2(2-4)}{2} y-\left(8+\frac{6(2-4)^{2}}{4}\right) t}\right)}{2\left(1+e^{x+\frac{(2-2)}{2} y+z-\left(1+\frac{\left.3(2-2)^{2}\right)}{4}+3 \frac{1}{1}\right) t}+e^{2 x+\frac{2(2-4)}{2} y-\left(8+\frac{\left.6(2-4)^{2}\right)}{4}\right) t}\right)} \\
=\frac{2 e^{2 x-14 t-2 y}+e^{x-5 t+z}}{e^{2 x-14 t-2 y}+e^{x-5 t+z}+1}=v_{x} \\
v=\ln \left(1+e^{2 x-14 t-2 y}+e^{x-5 t+z}\right)
\end{gathered}
$$

and simplifying the expression

$$
v_{x t}+6 \beta v_{x} v_{x x}+v_{x x x x}-\frac{3}{2} \alpha^{2} v_{x}^{2} v_{x x}+3 \sigma^{2} v_{y y}-3 \alpha \sigma v_{x x} v_{y}+3 \sigma^{2} v_{z z}
$$

we obtain

$$
\frac{6\left(e^{3 x-18 t-2 y+z}+2 e^{5 x-32 t-4 y+z}+e^{7 x-46 t-6 y+z}+e^{4 x-22 t-2 y+2 z}+e^{5 x-26 t-2 y+3 z}+2 e^{6 x-36 t-4 y+2 z}\right)}{\left(e^{2 x-14 t-2 y}+e^{x-4 t+z}+1\right)^{4}} .
$$

We used standard LaTex techniques to simplify the expression in the left hand side of (6). This expression is positive for any choice of $x, y, z$ and $t$. That is (14) is not the solution of the equation (6).

Now to find a correct double-kink solution we set

$$
\begin{aligned}
& v(x, y, z, t)=R\left(\ln \left(1+e^{k_{1} x+r_{1} y+s_{1} z+c_{1} t}+e^{k_{2} x+r_{2} y+s_{2} z+c_{2} t}\right),\right. \\
& v_{x}=R \frac{k_{1} e^{x k_{1}+\cdots}+k_{2} e^{x k_{2}+\cdots}}{1+e^{x k_{1}+\cdots}+e^{x k_{2}+\cdots}}, \\
& v_{x t}=R \frac{c_{1} k_{1} e^{x k_{1}+\cdots}+c_{2} k_{2} e^{x k_{2}+\cdots}+\sum_{i, j=1,2} c_{i} k_{j} e^{x k_{1}+\cdots} e^{x k_{2}+\cdots}}{\left(1+e^{x k_{1}+\cdots}+e^{x k_{2}+\cdots}\right)^{2}}, \\
& 6 \beta v_{x} v_{x x}=6 \beta R^{2} \frac{k_{1} e^{x k_{1}+\cdots}+k_{2} e^{x k_{2}+\cdots}}{1+e^{x k_{1}+\cdots}+e^{x k_{2}+\cdots}} \\
& \frac{k_{1}^{2} e^{x k_{1}+\cdots}+k_{2}{ }^{2} e^{x k_{2}+\cdots}+k_{1}{ }^{2} e^{x k_{1}+\cdots} e^{x k_{2}+\cdots}+k_{2}{ }^{2} e^{x k_{1}+\cdots} e^{x k_{2}+\cdots}-2 k_{1} k_{2} e^{x k_{1}+\cdots} e^{x k_{2}+\cdots}}{\left(1+e^{x k_{1}+\cdots}+e^{x k_{2}+\cdots}\right)^{2}}, \\
& v_{x x x x}=\frac{R}{\left(1+e^{x k_{1}+\cdots}+e^{x k_{2}+\cdots}\right)^{4}}\left(k_{1}{ }^{4} e^{x k_{1}+\cdots}-4 k_{1}{ }^{4} e^{2 x k_{1}+\cdots}+k_{2}{ }^{4} e^{x k_{2}+\cdots}\right. \\
& +k_{1}{ }^{4} e^{3 x k_{1}+\cdots}-4 k_{2}{ }^{4} e^{2 x k_{2}+\cdots}+k_{2}{ }^{4} e^{3 x k_{2}+\cdots}+3 k_{1}{ }^{4} e^{x k_{1}+\cdots} e^{x k_{2}+\cdots}+3 k_{2}{ }^{4} e^{x k_{1}+\cdots} e^{x k_{2}+\cdots} \\
& +3 k_{1}^{4} e^{x k_{1}+\cdots} e^{2 x k_{2}+\cdots}-8 k_{1}^{4} e^{x k_{2}+\cdots} e^{2 x k_{1}+\cdots}+k_{1}^{4} e^{x k_{1}+\cdots} e^{3 x k_{2}+\cdots}+k_{1}^{4} e^{x k_{2}+\cdots} e^{3 x k_{1}+\cdots} \\
& -8 k_{2}^{4} e^{x k_{1}+\cdots} e^{2 x k_{2}+\cdots}+3 k_{2}^{4} e^{x k_{2}+\cdots} e^{2 x k_{1}+\cdots}-4 k_{1}^{4} e^{2 x k_{1}+\cdots} e^{2 x k_{2}+\cdots}+k_{2}^{4} e^{3 x k_{2}+\cdots} e^{x k_{1}+\cdots} \\
& +k_{2}^{4} e^{3 x k_{1}+\cdots} e^{x k_{2}+\cdots}-4 k_{2}^{4} e^{2 x k_{2}+\cdots} e^{2 x k_{1}+\cdots}-4 k_{1} k_{2}^{3} e^{x k_{1}+\cdots} e^{x k_{2}+\cdots} \\
& -4 k_{1}^{3} k_{2} e^{x k_{1}+\cdots} e^{x k_{2}+\cdots}+16 k_{2}^{3} k_{1} e^{2 x k_{2}+\cdots} e^{x k_{1}+\cdots}-8 k_{1} k_{2}^{3} e^{2 x k_{1}+\cdots} e^{x k_{2}+\cdots} \\
& -8 k_{1}^{3} k_{2} e^{x k_{1}+\cdots} e^{2 x k_{2}+\cdots}+16 k_{1}^{3} k_{2} e^{x k_{2}+\cdots} e^{2 x k_{1}+\cdots}-4 k_{1} k_{2}^{3} e^{x k_{1}+\cdots} e^{3 x k_{2}+\cdots} \\
& -4 k_{2}^{3} k_{1} e^{3 x k_{1}+\cdots} e^{x k_{2}+\cdots}-4 k_{1}^{3} k_{2} e^{3 x k_{2}+\cdots} e^{x k_{1}+\cdots}-4 k_{2} k_{1}^{3} e^{3 x k_{1}+\cdots} e^{x k_{2}+\cdots} \\
& +16 k_{2}^{3} k_{1} e^{2 x k_{1}+\cdots} e^{2 x k_{2}+\cdots}+16 k_{1}^{3} k_{2} e^{2 x k_{2}+\cdots} e^{2 x k_{1}+\cdots}-6 k_{1}^{2} k_{2}^{2} e^{x k_{1}+\cdots} e^{x k_{2}+\cdots} \\
& \left.+6 k_{1}^{2} k_{2}^{2} e^{x k_{1}+\cdots} e^{3 x k_{2}+\cdots}+6 k_{1}^{2} k_{2}^{2} e^{x k_{2}+\cdots} e^{3 x k_{1}+\cdots}-24 k_{1}^{2} k_{2}^{2} e^{2 x k_{1}+\cdots} e^{2 x k_{2}+\cdots}\right) \text {, } \\
& -\frac{3}{2} \alpha^{2} v_{x}^{2} v_{x x}=-\frac{2}{3} \alpha^{2} R^{3} \frac{\left(k_{1} e^{x k_{1}+\cdots}+k_{2} e^{x k_{2}+\cdots}\right)^{2}}{\left(1+e^{x k_{1}+\cdots}+e^{x k_{2}+\cdots}\right)^{2}} \\
& \frac{k_{1}^{2} e^{x k_{1}+\cdots}+k_{2}^{2} e^{x k_{2}+\cdots}+k_{1}^{2} e^{x k_{1}+\cdots} e^{x k_{2}+\cdots}+k_{2}^{2} e^{x k_{1}+\cdots} e^{x k_{2}+\cdots}-2 k_{1} k_{2} e^{x k_{1}+\cdots} e^{x k_{2}+\cdots}}{\left(1+e^{x k_{1}+\cdots}+e^{x k_{2}+\cdots}\right)^{2}}, \\
& 3 \sigma^{2} v_{y y}=3 \sigma^{2} R \frac{r_{1}^{2} e^{x k_{1}+\cdots}+r_{2}^{2} e^{x k_{2}+\cdots}+r_{1}^{2} e^{x k_{1}+\cdots} e^{x k_{2}+\cdots}+r_{2}^{2} e^{x k_{1}+\cdots} e^{x k_{2}+\cdots}-2 r_{1} r_{2} e^{x k_{1}+\cdots} e^{x k_{2}+\cdots}}{\left(1+e^{x k_{1}+\cdots}+e^{x k_{2}+\cdots}\right)^{2}},
\end{aligned}
$$




$$
\begin{gathered}
-3 \alpha \sigma v_{x x} v_{y}=-3 \alpha \sigma R^{2} \frac{k_{1}^{2} e^{x k_{1}+\cdots}+k_{2}^{2} e^{x k_{2}+\cdots}+k_{1}^{2} e^{x k_{1}+\cdots} e^{x k_{2}+\cdots}+k_{2}^{2} e^{x k_{1}+\cdots} e^{x k_{2}+\cdots}-2 k_{1} k_{2} e^{x k_{1}+\cdots} e^{x k_{2}+\cdots}}{\left(1+e^{x k_{1}+\cdots}+e^{x k_{2}+\cdots}\right)^{2}} \\
\frac{r_{1} e^{x k_{1}+\cdots}+r_{2} e^{x k_{2}+\cdots}}{1+e^{x k_{1}+\cdots}+e^{x k_{2}+\cdots}}, \\
3 \sigma^{2} v_{z z}=3 \sigma^{2} R \frac{s_{1}^{2} e^{x k_{1}+\cdots}+s_{2}^{2} e^{x k_{2}+\cdots+}+k_{1}^{2} e^{x k_{1}+\cdots} e^{x k_{2}+\cdots}+s_{2}^{2} e^{x k_{1}+\cdots} e^{x k_{2}+\cdots}-2 s_{1} s_{2} e^{x k_{1}+\cdots} e^{x k_{2}+\cdots}}{\left(1+e^{\left.x k_{1}+\cdots+e^{x k_{2}+\cdots}\right)^{2}}\right.} .
\end{gathered}
$$

Substituting into (6), multiplying by $\left(1+e^{x k_{1}+\cdots}+e^{x k_{2}+\cdots}\right)^{4}$ and equalizing the coefficients of $e^{m x k_{1}+\cdots}$ and $e^{n x k_{2}+\cdots}, m=1,2$, 3, we obtain the equations

$$
\begin{gathered}
k_{1}^{4}+3 \sigma^{2} r_{1}^{2}+3 \sigma^{2} s_{1}^{2}+c_{1} k_{1}=0, \\
k_{2}^{4}+3 \sigma^{2} r_{2}^{2}+3 \sigma^{2} s_{2}^{2}+c_{2} k_{2}=0, \\
2 k_{1}^{2}-2 R \beta k_{1}+R \alpha \sigma r_{1}=0 \\
2 k_{2}^{2}-2 R \beta k_{2}+R \alpha \sigma r_{2}=0 \\
-4 \beta k_{1}+2 \alpha \sigma r_{1}+R \alpha^{2} k_{1}^{2}=0, \\
-4 \beta k_{2}+2 \alpha \sigma r_{2}+R \alpha^{2} k_{2}^{2}=0,
\end{gathered}
$$

which gives

$$
R=\frac{2}{\alpha}, \quad r_{1}=\frac{1}{\alpha \sigma}\left(2 \beta k_{1}-\alpha k_{1}^{2}\right), \quad r_{2}=\frac{1}{\alpha \sigma}\left(2 \beta k_{2}-\alpha k_{2}^{2}\right) .
$$

Coefficients of mixed products $e^{m x k_{1}+\cdots} e^{n x k_{2}+\cdots}$ can be translated into the equation

$$
\left(k_{1}-k_{2}\right)^{4}+3 \sigma^{2}\left(r_{1}-r_{2}\right)^{2}+3 \sigma^{2}\left(s_{1}-s_{2}\right)^{2}+\left(c_{1}-c_{2}\right)\left(k_{1}-k_{2}\right)=0,
$$

and we obtain the next system for unknowns $s_{2}$ and $c_{2}$ (for given $k_{1}, s_{1}, r_{1}=\frac{1}{\alpha \sigma}\left(2 \beta k_{1}-\alpha k_{1}^{2}\right), r_{2}=\frac{1}{\alpha \sigma}\left(2 \beta k_{2}-\alpha k_{2}^{2}\right)$, $\left.c_{1}=-k_{1}^{3}-3 \frac{\sigma^{2}}{k_{1}} r_{1}^{2}-3 \frac{\sigma^{2}}{k_{1}} s_{1}^{2}, k_{2}\right)$

$$
\begin{gathered}
k_{1}^{4}+3 \sigma^{2}\left(r_{1}^{2}+s_{1}^{2}\right)+c_{1} k_{1}=0 \\
k_{2}^{4}+3 \sigma^{2}\left(r_{2}^{2}+s_{2}^{2}\right)+c_{2} k_{2}=0 \\
\left(k_{1}-k_{2}\right)^{4}+3 \sigma^{2}\left(r_{1}-r_{2}\right)^{2}+3 \sigma^{2}\left(s_{1}-s_{2}\right)^{2}+\left(c_{1}-c_{2}\right)\left(k_{1}-k_{2}\right)=0
\end{gathered}
$$

or

$$
\begin{gathered}
c_{1}=-k_{1}^{3}-3 \frac{\sigma^{2}}{k_{1}} r_{1}^{2}-3 \frac{\sigma^{2}}{k_{1}} s_{1}^{2} \\
c_{2}=-k_{2}^{3}-3 \frac{\sigma^{2}}{k_{2}} r_{2}^{2}-3 \frac{\sigma^{2}}{k_{2}} s_{2}^{2} \\
c_{1}-c_{2}=-\left(k_{1}-k_{2}\right)^{3}-3 \sigma^{2} \frac{\left(r_{1}-r_{2}\right)^{2}+\left(s_{1}-s_{2}\right)^{4}}{k_{1}-k_{2}} .
\end{gathered}
$$

First and second equations replaced into the third one gives

$$
\begin{gathered}
-k_{1}^{3}-3 \frac{\sigma^{2}}{k_{1}} r_{1}^{2}-3 \frac{\sigma^{2}}{k_{1}} s_{1}^{2}-\left(-k_{2}^{3}-3 \frac{\sigma^{2}}{k_{2}} r_{2}^{2}-3 \frac{\sigma^{2}}{k_{2}} s_{2}^{2}\right) \\
=-\left(k_{1}-k_{2}\right)^{3}-3 \sigma^{2} \frac{\left(r_{1}-r_{2}\right)^{2}+\left(s_{1}-s_{2}\right)^{4}}{k_{1}-k_{2}}
\end{gathered}
$$

and

$$
\left(\sigma^{2}\left(k_{1} r_{2}-k_{2} r_{1}\right)^{2}+\sigma^{2}\left(k_{1} s_{2}-k_{2} s_{1}\right)^{2}-k_{1}^{2} k_{2}^{2}\left(k_{1}-k_{2}\right)^{2}\right)=0
$$

Now substituting

$$
r_{i}=\frac{1}{\alpha \sigma}\left(2 \beta k_{i}-\alpha k_{i}^{2}\right), \quad i=1,2
$$

we have 


$$
\begin{gathered}
\sigma^{2}\left(k_{1} \frac{1}{\alpha \sigma}\left(2 \beta k_{2}-\alpha k_{2}^{2}\right)-k_{2} \frac{1}{\alpha \sigma}\left(2 \beta k_{1}-\alpha k_{1}^{2}\right)\right)^{2} \\
+\sigma^{2}\left(k_{1} s_{2}-k_{2} s_{1}\right)^{2}-k_{1}^{2} k_{2}^{2}\left(k_{1}-k_{2}\right)^{2} \\
=\sigma^{2}\left(k_{1} s_{2}-k_{2} s_{1}\right)^{2}
\end{gathered}
$$

and

$$
s_{2}=\frac{k_{2}}{k_{1}} s_{1} .
$$

This relationship allows us to find the elegant double-kink solutions. First, we find $c_{2}$ from the second equation in (21) and (25):

$$
c_{2}=-k_{2}^{3}-3 \frac{\sigma^{2}}{k_{2}} r_{2}^{2}-3 \frac{\sigma^{2} k_{2}}{k_{1}} s_{1}^{2}
$$

and therefore, the exact solution is

$$
\begin{gathered}
v=\frac{2}{\alpha} \ln \left(1+e^{k_{1} x+\frac{2 \beta k_{1}-\alpha k_{1}^{2}}{\alpha \sigma} y+s_{1} z-\left(k_{1}^{3}+3 \frac{\left(2 \beta k_{1}-\alpha k_{1}^{2}\right)^{2}}{\alpha^{2} k_{1}}+3 \frac{\sigma^{2} s_{1}^{2}}{k_{1}}\right) t}\right. \\
+e^{k_{2} x+\frac{2 \beta k_{2}-\alpha k_{2}^{2}}{\alpha \sigma} y+\frac{k_{2}}{k_{1}} s_{1} z-\left(k_{2}^{3}+3 \frac{\left(2 \beta k_{2}-\alpha k_{2}^{2}\right)^{2}}{\alpha^{2} k_{2}}+3 \frac{\sigma^{2} k_{2} s_{1}^{2}}{k_{1}^{2}}\right) t} \\
=\frac{2}{\alpha} \ln \left(1+\sum_{i=1}^{2} e^{k_{i} x+\frac{2 \beta k_{i}-\alpha k_{i}^{2}}{\alpha \sigma} y+\frac{k_{i}}{k_{1}} s_{1} z-\left(k_{i}^{3}+3 \frac{\left(2 \beta k_{i}-\alpha k_{i}^{2}\right)^{2}}{\alpha^{2} k_{1}}+3 \frac{\sigma^{2} k_{i} s_{1}^{2}}{k_{1}^{2}}\right) t}\right)
\end{gathered}
$$

Now to find a wave travelling solution of the equation (2), we take $k_{1}=n_{1}+i n_{2}, k_{2}=n_{1}-i n_{2}, s_{1}=s+\frac{n_{2}}{n_{1}} s i$, $n_{1}, n_{2}, s \in R$ and consider the function

We have for $i=1$

$$
\frac{2}{\alpha} \ln \left(1+\sum_{i=1}^{2} e^{k_{i} x+\frac{2 \beta k_{i}-\alpha k_{i}^{2}}{\alpha \sigma} y+\frac{k_{i}}{k_{1}} s_{1} z-\left(k_{i}^{3}+3 \frac{\left(2 \beta k_{i}-\alpha k_{i}^{2}\right)^{2}}{\alpha^{2} k_{1}}+3 \frac{\sigma^{2} k_{i} s_{1}^{2}}{k_{1}^{2}}\right) t}\right) .
$$

$$
\begin{aligned}
& k_{1} x+\frac{2 \beta k_{1}-\alpha k_{1}^{2}}{\alpha \sigma} y+s_{1} z-\left(k_{1}^{3}+3 \frac{\left(2 \beta k_{1}-\alpha k_{1}^{2}\right)^{2}}{\alpha^{2} k_{1}}+3 \frac{\sigma^{2} s_{1}^{2}}{k_{1}}\right) t \\
& \left(n_{1}+i n_{2}\right) x+\frac{2 \beta\left(n_{1}+i n_{2}\right)-\alpha\left(n_{1}+i n_{2}\right)^{2}}{\alpha \sigma} y+s\left(1+\frac{n_{2}}{n_{1}} i\right) z \\
& -\left(\left(n_{1}+i n_{2}\right)^{3}+3 \frac{\left(2 \beta\left(n_{1}+i n_{2}\right)-\alpha\left(n_{1}+i n_{2}\right)^{2}\right)^{2}}{\alpha^{2}\left(n_{1}+i n_{2}\right)}+\frac{3 \sigma^{2} s^{2}\left(1+\frac{n_{2}}{n_{1}} i\right)^{2}}{\left(n_{1}+i n_{2}\right)}\right) t \\
& =n_{1} x+i n_{2} x+\frac{2 \beta n_{1}+\alpha n_{2}^{2}-\alpha n_{1}^{2}}{\alpha \sigma} y+\frac{n_{2}\left(2 \beta-2 \alpha n_{1}\right)}{\alpha \sigma} y i+s\left(1+\frac{n_{2}}{n_{1}} i\right) z \\
& =-\frac{1}{\alpha^{2} n_{1}}\left(3 s^{2} \alpha^{2} \sigma^{2}+4 \alpha^{2} n_{1}^{4}-12 \alpha^{2} n_{1}^{2} n_{2}^{2}-12 \alpha \beta n_{1}^{3}+12 \alpha \beta n_{1} n_{2}^{2}+12 \beta^{2} n_{1}^{2}\right) t \\
& -\frac{1}{\alpha^{2} n_{1}^{2}} n^{2}\left(3 s^{2} \alpha^{2} \sigma^{2}+12 \alpha^{2} n_{1}^{4}-4 \alpha^{2} n_{1}^{2} n_{2}^{2}-24 \alpha \beta n_{1}^{3}+12 \beta^{2} n_{1}^{2}\right) t i,
\end{aligned}
$$

and for $i=2$

$$
\begin{gathered}
k_{2} x+\frac{2 \beta k_{2}-\alpha k_{2}^{2}}{\alpha \sigma} y+\frac{k_{2} s_{1}}{k_{1}} z-\left(k_{2}^{3}+3 \frac{\left(2 \beta k_{2}-\alpha k_{2}^{2}\right)^{2}}{\alpha^{2} k_{2}}+3 \frac{k_{2} \sigma^{2}}{k_{1}^{2}} s_{1}^{2}\right) t \\
=\left(n_{1}-i n_{2}\right) x+\frac{2 \beta\left(n_{1}-i n_{2}\right)-\alpha\left(n_{1}-i n_{2}\right)^{2}}{\alpha \sigma} y+\frac{\left(n_{1}-i n_{2}\right) s\left(1+\frac{n_{2}}{n_{1}} i\right)}{n_{1}+i n_{2}} z \\
-\left(\left(n_{1}-i n_{2}\right)^{3}+\frac{3\left(2 \beta\left(n_{1}-i n_{2}\right)-\alpha\left(n_{1}-i n_{2}\right)^{2}\right)^{2}}{\alpha^{2}\left(n_{1}-i n_{2}\right)}\right.
\end{gathered}
$$




$$
\begin{gathered}
+\frac{3\left(n_{1}-i n_{2}\right) \sigma^{2} s^{2}\left(1+\left(n_{2} / n_{1}\right) i\right)^{2}}{\left(n_{1}+i n_{2}\right)^{2}} t \\
=n_{1} x-i n_{2} x+\frac{2 \beta n_{1}+\alpha n_{2}^{2}-\alpha n_{1}^{2}}{\alpha \sigma} y-\frac{n_{2}\left(2 \beta-2 \alpha n_{1}\right)}{\alpha \sigma} y i \\
-\frac{1}{\alpha^{2} n_{1}}\left(3 s^{2} \alpha^{2} \sigma^{2}+4 \alpha^{2} n_{1}^{4}-12 \alpha_{1}{ }^{2} n_{1}{ }^{2} n_{2}^{2}-12 \alpha \beta n_{1}^{3}+12 \alpha \beta n_{1} n_{2}^{2}+12 \beta^{2} n_{1}^{2}\right) t \\
+\frac{n^{2}}{\alpha^{2} n_{1}^{2}}\left(3 s^{2} \alpha^{2} \sigma^{2}+12 \alpha^{2} n_{1}^{4}-4 \alpha^{2} n_{1}^{2} n_{2}^{2}-24 \alpha \beta n_{1}^{3}+12 \beta^{2} n_{1}^{2}\right) t i .
\end{gathered}
$$

This in turn gives

$$
\begin{gathered}
\sum_{n=1}^{2} e^{k_{i} x+\frac{2 \beta k_{i}-\alpha k_{i}^{2}}{\alpha \sigma} y+\frac{k_{i} s_{1}}{k_{1}} z-\left(k_{i}^{3}+3 \frac{\left(2 \beta k_{i}-\alpha k_{i}^{2}\right)^{2}}{k_{i} \alpha^{2}}+3 \frac{k_{i} \sigma^{2}}{k_{1}^{2}} s_{1}^{2}\right) t} \\
=2 \cos \left(n_{1} x+\frac{2 \beta n_{1}+\alpha n_{2}^{2}-\alpha n_{1}^{2}}{\alpha \sigma} y+c z\right. \\
-\frac{1}{\alpha^{2} n_{1}}\left(3 s^{2} \alpha^{2} \sigma^{2}+4 \alpha^{2} n_{1}^{4}-12 \alpha^{2} n_{1}^{2} n_{2}^{2}-12 \alpha \beta n_{1}^{3}+12 \alpha \beta n_{1} n_{2}^{2}+12 \beta^{2} n_{1}^{2}\right) t \\
=2 \cos \left(n_{1} x+\frac{2 \beta n_{1}+\alpha n_{2}^{2}-\alpha n_{1}^{2}}{\alpha \sigma} y+c z\right. \\
\left.-12 t\left(\frac{\beta^{2} n_{1}}{\alpha^{2}}+\frac{n_{1}^{3}}{3}+\frac{\sigma^{2} s^{2}}{4 n^{1}}-\frac{\beta}{\alpha} n_{1}^{2}-n_{2}^{2} n_{1}-\frac{\beta}{\alpha} n_{2}^{2}\right)\right)
\end{gathered}
$$

and the travelling-wave solution

where

$$
u(x, y, z, t)=\frac{4 n_{1} \sin \left(n_{1} x+\phi\left(n_{1}, n_{2}\right) y+s z-\psi\left(n_{1}, n_{2}, s\right) t\right)}{\alpha\left(1+2 \cos \left(n_{1} x+\phi\left(n_{1}, n_{2}\right) y+s z-\psi\left(n_{1}, n_{2}, s\right) t\right)\right.}
$$

$$
\begin{gathered}
\phi\left(n_{1}, n_{2}\right) \equiv \frac{2 \beta n_{1}-\alpha n_{1}^{2}+\alpha n_{2}^{2}}{\alpha \sigma}, \\
\psi\left(n_{1}, n_{2}, s\right) \equiv 12\left(\frac{\beta^{2} n_{1}}{\alpha^{2}}+\frac{n_{1}^{3}}{3}+\frac{\sigma^{2} s}{4 n_{1}}-\frac{\beta}{\alpha} n_{1}^{2}-n_{2}^{2} n_{1}-\frac{\beta}{\alpha} n_{2}^{2}\right) .
\end{gathered}
$$

\section{Conclusions}

This paper has described how the travelling-wave solutions can be received from double-kink solutions. We found the necessary conditions for double kink solution to exist. The dispersion relations were also derived.

\section{References}

[1] Osborne, A. (2010). Nonlinear ocean waves and inverse scattering transform, Elsevier, Amsterdam, 2010.

[2] Konopelchenko, B. G. (1991). Inverse spectral transform for the (2+1)-dimensional Gardner equation, Inverse Problems, V. 7, 1991, pp. 739-753.

[3] Konopelchenko, B. G., Dubrovsky, V. G. (1983). On the general structure of nonlinear equations integrable by the general linear spectral problem, Phys. Lett. A, V.95, N.9, 1983, pp. 457-461.

[4] Yu, G.-F., Tam, H.-W. (2007). On the (2+1)—dimensional Gardner equation: Determinant solutions and pfaffianization, J. Math. Anal. Appl., V.330, 2007, pp. 989-1001.

[5] Wazwaz, A. M. (2007). New solitons and kink solutions for the Gardner equation, Commun. Nonlin. Sci. Numer. Simulat., V.12, N.8, 2007, pp. 1395-1404.

[6] Wazwaz, A. M. (2008). Solitons and singular solitons for the Gardner-KP equation, Appl. Math. Comput., V.204, N.1, 2008, pp. 162-169.

[7] Wazwaz, A. M. (2014). Multiple kink solutions for the (2+1)-dimensional integrable Gardner equation, Proc. Romanian Acad. A, V.15, 2014, pp. 241-246. 
[8] Naz, R., Ali, Z., Naeem, I. (2013). Reductions and new exact solutions of ZK, Gardner KP, and modified KP Equations via generalized double reduction theorem, Abstract and Applied Analysis, 2013, 340564.

[9] Cai, P., Tang, J.-S., Li, Z.-B. Bifurcation of exact traveling wave solutions for Gardner and Gardner--KP equations, Intern. J. Appl. Math. Statistics, V.44, N.14, pp. 461-468.

[10] Hirota, R. (2004). The Direct Method in Soliton Theory, Cambridge University Press, Cambridge, 2004.

[11] Wazwaz, A. M. (2017). Two (3+1)-dimensional Gardner-type equation with multiple kink solutions, Rom. Rep. Phys., V.69, N.108, 2017.

[12] Porubov, A. V., Maugin, G. A., Andrievsky, B. R. (2011). Wave Motion, doi: 10.1016/j.wavemoti. 2011.04.012. 2011.

[13] Vassilev, V. M., Djongjorov, P. A., Hadzhilazova, M. Ts., Mladenov, I. M. (2011). Traveling Wave Solutions of the Gardner Equation and Motion of Plane Curves Governed by the mKdV Flow, AIP Conf. Proc., V.1404, N.86, 2011. doi: 10.1063/1.3659907. 